\title{
Stilt: Easy Emulation of Time Series AR(1) Computer Model Output in Multidimensional Parameter Space
}

\author{
by Roman Olson, Kelsey L. Ruckert, Won Chang, Klaus Keller, Murali Haran, and Soon-Il An
}

\begin{abstract}
Statistically approximating or "emulating" time series model output in parameter space is a common problem in climate science and other fields. There are many packages for spatio-temporal modeling. However, they often lack focus on time series, and exhibit statistical complexity. Here, we present the R package stilt designed for simplified AR(1) time series Gaussian process emulation, and provide examples relevant to climate modelling. Notably absent is Markov chain Monte Carlo estimation - a challenging concept to many scientists. We keep the number of user choices to a minimum. Hence, the package can be useful pedagogically, while still applicable to real life emulation problems. We provide functions for emulator cross-validation, empirical coverage, prediction, as well as response surface plotting. While the examples focus on climate model emulation, the emulator is general and can be also used for kriging spatio-temporal data.
\end{abstract}

\section{Introduction}

Emulation of computer model behavior in parameter space is a challenging problem (Drignei et al., 2008; Higdon et al., 2008; Holden et al., 2010; Bhat et al., 2012; Olson et al., 2012; Sexton et al., 2012; Olson et al., 2013; Chang et al., 2014a,b). Often a limited number of runs are available and we desire to estimate model output at a variety of new parameter settings (Drignei et al., 2008; Holden et al., 2010; Bhat et al., 2012; Olson et al., 2012; Sexton et al., 2012; Olson et al., 2013; Chang et al., 2014b). Emulation is often used in the context of another common problem, input parameter estimation. Here, we desire to find probability distribution functions for model input parameters given observational data (e.g., Olson et al., 2012; Chang et al., 2014b). While model output is often multi-dimensional, we restrict the discussion here to time series although our approach can apply to vector output more generally.

Gaussian processes (Kennedy and O'Hagan, 2001; Rasmussen and Williams, 2006; Higdon et al., 2008; Rougier, 2008) are a very useful methodology for such emulation. Gaussian processes provide the best linear unbiased prediction under highly general conditions (Stein, 1999). This methodology assumes that model response is a smooth function of parameter settings. Central to the method is formulation of the covariance function, which quantifies the covariance between model output as a function of distance in each model input parameter. This covariance is typically parametrized by parameters controlling, for example, magnitude or the correlation ranges in each coordinate dimension. Associated with emulator parameters is a likelihood function given the model output. There are two main approaches: (i) find the "best" parameter setting that maximizes this likelihood, or (ii) find the full probability distribution of the parameters. The next step is prediction at new parameter settings. The former approach ignores the uncertainty in emulator parameters during the prediction, while the latter approach accounts for it. The prediction is probabilistic as it includes the predictive mean and the uncertainty around it.

There are many existing $\mathrm{R}$ packages to perform Gaussian process emulation for vector and time series output. In particular, they include gstat (Gräler et al., 2016), mlegp (Dancik and Dorman, 2008; Dancik, 2013), spBayes (Finley et al., 2015), ramps (Smith et al., 2008), spTimer (Bakar and Sahu, 2015a,b), and RandomFields (Schlather et al., 2015). These spatio-temporal packages are usually general and do not focus on time series specifically. Moreover, they are usually presented in context of spatial interpolation ("kriging") rather than emulation. They may provide flexible functionality in terms of covariance functions (RandomFields), handling replicates (mlegp), dealing with missing observations (spTimer), or considering both areal as well as point observations (ramps). The procedure for fitting emulator parameters differs between packages. Some packages use maximum likelihood or another form of optimization to find the best parameters, while others use Bayesian analysis.

Due to their statistical complexity, the broad scientific community may find it challenging to use existing software. Particularly, the concept of Markov chain Monte Carlo (MCMC) employed in some work may be unfamiliar to some scientists. In addition, a large variety of modeling or prior choices may leave a user struggling with which model or prior they should employ and why. Many packages appear to be tailored to a statistical audience, and elaborate statistical terminology may be beyond the grasp for some scientists. Second, the packages do not usually focus on the spatio-temporal output ubiquitous in computer modelling. Thus, scientists may invest substantial time in finding the right 
function among the plethora available. Finally, there is an issue of terminology: many scientists may not realize that "spatio-temporal modeling" may disguise the same technique as "emulation" and hence, may be oblivious to the applicability of these packages.

We introduce the $R$ package stilt version 1.3.0 for simplified Gaussian Process AR(1) time series emulation with a focus on climate modeling (Olson et al., 2017). The package is available on Comprehensive R Archive Network (CRAN). The main differences from prior approaches are: (i) a simplified framework with fewer modeling choices and no MCMC that is still applicable to real-life problems, and (ii) a focus on emulation of time-series in parameter space. Specifically, there is only one function to fit the emulator to model output and only one function for prediction. Statistical modeling accounts for random noise in the model output through the nugget term, and also allows for user-chosen linear terms in model parameters and/or time. Mathematically, this is the first public implementation of a separable covariance model of Rougier (2008). In this model, space-time covariance between two locations is a product of a space covariance and a time covariance term. This allows the use of matrix algebra to achieve considerable computational gains. We include useful utility functions for extensive cross-validation, 2D response surface plotting, and empirical $95 \%$ prediction interval coverage. In three examples, we apply stilt to emulation in spaces ranging from one- to five-dimensional. Note that whilst the software is for emulation of time series output in parameter space, the package is general and can be used for interpolation of observations (or model output) in geographical coordinates (e.g., Cressie and Johannesson, 2008; Jones et al., 2009; Hansen et al., 2010; Bhat et al., 2012; Hirahara et al., 2014).

In the rest of the paper we first outline the statistical approach, then give three examples in 1D, $2 \mathrm{D}$, and $5 \mathrm{D}$ parameter space, then present some concluding remarks. We provide technical details in the Appendix.

\section{Statistical approach}

\section{Overview of Approach}

Here, we briefly describe our statistical approach. Our statistical model includes optional linear terms in parameters and/or time, a smooth Gaussian process, and a purely random nugget term. The Gaussian process represents smooth non-linear effects of parameters or time on the model output. The nugget term represents purely random effects. For climate model output, this corresponds to internal climate variability.

The first step is fitting the Gaussian process statistical model to the computer model output. Specifically, the statistical model has several parameters. They control the linear slopes in parameters and time, the correlation ranges of the field in each of the input parameters, the temporal autocorrelation, the overall magnitude of the covariance, and the nugget strength. Associated with the emulator parameters is the likelihood which quantifies how likely the emulator parameter values are given the model output. During the emulator fitting, we vary the parameters so that this likelihood is maximized to obtain the optimized emulator. We use this optimized emulator for prediction. The prediction follows the standard Gaussian process theory. The rest of the section describes the details of the statistical methodology.

\section{Statistical model}

We base our implementation on standard Gaussian process theory (Cressie, 1993; Stein, 1999; Rasmussen and Williams, 2006). Consider the case of interpolating spatio-temporal model output of a perturbed parameter model ensemble in parameter space. Let $y_{i, j} \in R$ be physical model output at a parameter setting $\theta_{i}$ and a time $t_{j}$. Time values form an $n$-dimensional regularly spaced vector $\boldsymbol{t}=\left(t_{1}, \ldots, t_{n}\right)^{T}$.

Each parameter setting is an $m$-dimensional vector: $\boldsymbol{\theta}_{\boldsymbol{i}}=\left(\theta_{1, i}, \ldots, \theta_{m, i}\right)$. For all $p$ model runs, the parameter settings $\boldsymbol{\theta}_{i}$ form a $p \times m$ parameter matrix $\Theta$. Let $\boldsymbol{y}_{j}=\left(y_{1, j}, \ldots, y_{p, j}\right)^{T}$ be a $p$-dimensional vector of model outputs for all $p$ parameters for time $t_{j}$. Consecutively, the stacked $p n \times 1$ column matrix of all model output for times from 1 through $n$ is $\mathbf{Y}=\left(\mathbf{y}_{\mathbf{1}}{ }^{T}, \ldots, \boldsymbol{y}_{\boldsymbol{n}}{ }^{T}\right)^{T}$. Associated with $\mathbf{Y}$ is the $p n \times(m+1)$ design matrix $\mathbf{D}$. Its columns represent parameters and time, whereas rows correspond 
to elements of $\mathbf{Y}$. We calculate the design matrix $\mathbf{D}$ as:

$$
\mathbf{D}=\left(\left[\begin{array}{c}
1 \\
1 \\
\vdots \\
1
\end{array}\right]_{n \times 1} \otimes \Theta \quad \mathbf{t} \otimes\left[\begin{array}{c}
1 \\
1 \\
\vdots \\
1
\end{array}\right]_{p \times 1}\right)
$$

where $\otimes$ is the Kronecker product. We model the output as a Gaussian process such that

$$
\mathbf{Y} \sim N\left(\boldsymbol{\mu}_{\beta}, \Sigma\left(\boldsymbol{\xi}_{y}\right)\right)
$$

where $\mu_{\beta}$ is a mean function that is either constant or linear in any combination of parameters and/or time, and $\xi_{y}$ is a vector of covariance matrix parameters. We assume that $\mu_{\beta}=X \beta$, where $\beta$ is a column vector of regression coefficients, and $\mathbf{X}$ is a matrix of covariates. It always includes a column of ones as its first column to represent the intercept. Depending on the number of regressors, it can additionally have corresponding columns of the design matrix $\mathbf{D}$. As an example, for a mean function that is linear in time, $\boldsymbol{\beta}$ has dimensions of $2 \times 1$, and $\mathbf{X}$ is $p n \times 2$ :

$$
\mathbf{X}=\left[\begin{array}{cc}
1 & t_{1} \\
1 & t_{2} \\
\vdots & \vdots \\
1 & t_{n}
\end{array}\right]_{n \times 2} \otimes\left[\begin{array}{c}
1 \\
1 \\
\vdots \\
1
\end{array}\right]_{p \times 1}
$$

Under the assumption of separability (see Rougier, 2008), we can represent the covariance matrix $\Sigma$ as a Kronecker product of a separate covariance matrix in time $\Sigma_{t}$ and in parameters $\Sigma_{\theta}$ :

$$
\Sigma=\Sigma_{t} \otimes \Sigma_{\theta}
$$

This means that the covariance between any two locations in time and parameter space is a product of the time covariance term, and the parameter covariance term. This is a different approach to speed up the computation, compared to performing singular value decomposition (SVD) of model output (Dancik and Dorman, 2008; Dancik, 2013; Higdon et al., 2008; Chang et al., 2014b). We assume that the time covariance matrix $\Sigma_{t}(n \times n)$ has an $\mathrm{AR}(1)$ structure. $\mathrm{AR}(1)$ dependence in time is a feature of many environmental processes (e.g., Hasselmann, 1976; Keller and McInerney, 2008; Olson et al., 2013). To avoid identifiability issues, we do not use any multipliers for this matrix. Specifically, its $(j, k)$ element is:

$$
\zeta_{t, j k}=\frac{\rho^{\left|t_{j}-t_{k}\right|}}{1-\rho^{2}}
$$

where $\rho$ is the lag- 1 autocorrelation parameter. We assume that the parameter covariance $\Sigma_{\theta}(p \times p)$ is squared exponential, as in the package mlegp (Dancik and Dorman, 2008; Dancik, 2013). The squared exponential covariance function is frequently used in computer model emulation, as computer model outputs can be often represented using a highly smooth Gaussian process. The $(i, j)$ element of $\Sigma_{\theta}$ is:

$$
\varsigma_{\theta, i j}=\kappa \exp \left(-\sum_{k=1}^{m} \frac{\left|\theta_{k, i}-\theta_{k, j}\right|^{2}}{\phi_{k}^{2}}\right)+\zeta 1(i=j)
$$

where $\kappa$ is the partial sill, $\zeta$ is the nugget, and $\phi_{k}$ is the range parameter for the $k^{\text {th }}$ model input parameter. The range parameters form a vector $\boldsymbol{\phi}=\phi_{1}, \ldots, \phi_{m}$. We construct the total covariance matrix $(n p \times n p)$ as:

$$
\Sigma=\left[\begin{array}{ccc}
\varsigma_{t, 11} \Sigma_{\theta} & \cdots & \varsigma_{t, 1 n} \Sigma_{\theta} \\
\vdots & \ddots & \vdots \\
\zeta_{t, n 1} \Sigma_{\theta} & \cdots & \varsigma_{t, n n} \Sigma_{\theta}
\end{array}\right]
$$

Hence, the covariance parameters are $\xi_{y}=(\rho, \kappa, \phi, \zeta)^{T}$. The emulator parameters are $\psi=$ $\left(\beta^{T}, \xi_{y}{ }^{T}\right)^{T}$. The actual number of emulator parameters will be different depending on the number of model parameters that the ensemble varies and on the number of covariates. 


\section{Estimating emulator parameters}

We can write the log-likelihood for the model output $\mathbf{Y}$ given the emulator parameters $\psi$ as (see, e.g., Rasmussen and Williams, 2006):

$$
\ln L(\mathbf{Y} \mid \psi)=-\frac{1}{2}\left(\mathbf{Y}-\mu_{\beta}\right)^{T} \Sigma^{-1}\left(\mathbf{Y}-\mu_{\beta}\right)-\frac{1}{2} \ln |\Sigma|-\frac{n p}{2} \ln 2 \pi .
$$

Under the uniform priors for the emulator parameters,

$$
L(\boldsymbol{\psi} \mid \mathbf{Y}) \propto L(\mathbf{Y} \mid \psi) .
$$

Consequently, maximizing the log-likelihood for the model output also maximizes the likelihood for the parameters. Note that this likelihood evaluation involves a computationally expensive inverse of an $n p \times n p$ matrix. However, we can reduce the dimension of the inverted matrices to $p \times p$ and $n \times n$, and speed up the computation (see Appendix). Thus, the computational cost of inversion becomes $O\left(p^{3}\right)$ or $O\left(n^{3}\right)$, as opposed to $O\left([p n]^{3}\right)$. Further computational savings accrue when calculating $|\Sigma|$, considering that $\Sigma$ is a Kronecker product of two positive definite matrices (see Appendix).

We optimize the emulator parameters $\psi$ by maximizing the likelihood function over a reasonable parameter range using a local optimization routine. Specifically, stilt uses the nlminb function which calls FORTRAN code (Gay, 1990). In the package, there is an option to either fix $\beta$ parameters at their multiple linear regression estimates or to optimize them along with other emulator parameters.

\section{Prediction}

We are interested in predicting model output for all times for a new parameter setting $\theta^{*}$. We denote the $n$-dimensional vector output as $y^{*}=\left(y_{\theta^{*}, 1}, \ldots, y_{\theta^{*}, n}\right)^{T}$. Associated with the prediction parameter setting is an $n \times 1$ prediction design matrix $\mathbf{D}^{*}$ and a matrix of covariates $\mathbf{X}^{*}$ evaluated at predictions points. It is constructed similarly to $\mathbf{X}$. For a mean function that is linear in time, $\mathbf{X}^{*}$ is:

$$
\mathbf{X}^{*}=\left[\begin{array}{cc}
1 & t_{1} \\
1 & t_{2} \\
\vdots & \vdots \\
1 & t_{n}
\end{array}\right]_{n \times 2}
$$
2006):

The prediction is given by the following multivariate normal distribution (Rasmussen and Williams,

Here,

$$
y^{*} \sim N\left(\mu_{\beta}^{*}, \Sigma^{*}\right)
$$

$$
\mu_{\beta}^{*}=\mathbf{X}^{*} \boldsymbol{\beta}+\left(\Sigma_{t} \otimes \Sigma_{\theta^{*} \theta}\right) \Sigma^{-1}\left(\mathbf{Y}-\mu_{\beta}\right),
$$

where $\Sigma_{\theta^{*} \theta}$ is a $1 \times p$ cross-covariance matrix between the prediction parameter setting and all the ensemble parameters settings. For this matrix, we use the same covariance function as for $\Sigma_{\theta}$. To evaluate the mean function, we need the inverse of the $\Sigma$ matrix $(n p \times n p)$. However, using matrix algebra (Golub and Van Loan, 1996; Rougier, 2008), we can reduce the dimension to $p \times p$ by writing $\mu_{\beta}^{*}$ in the following way:

$$
\boldsymbol{\mu}_{\boldsymbol{\beta}}^{*}=\mathbf{X}^{*} \boldsymbol{\beta}+\left(\mathbf{I}_{n \times n} \otimes \Sigma_{\theta^{*} \theta} \Sigma_{\theta}^{-1}\right)\left(\mathbf{Y}-\boldsymbol{\mu}_{\boldsymbol{\beta}}\right) .
$$

The predictive covariance also requires an inversion of a $p \times p$ matrix only:

$$
\Sigma^{*}=(\kappa+\zeta) \Sigma_{t}-\Sigma_{t} \otimes \Sigma_{\theta^{*} \theta} \Sigma_{\theta}^{-1} \Sigma_{\theta^{*} \theta}^{T} .
$$

Prediction for many points uses the same inverse parameter covariance. Hence, we can calculate the inverse once and recycle it for prediction at many points. This further enhances computational savings.

\section{Examples using stilt}

\section{Preparing model output}

Here, we describe how to prepare model output for use with the stilt emulator. Two R list objects are required. These objects can be easily prepared by the user before the start of the analysis. The first, mpars, contains information on parameter settings in the ensemble. It has the following components: 
- par is the actual matrix of parameter settings, with rows corresponding to parameter index, and columns to the model run index.

- parnames is a vector of parameter names corresponding to the rows of par,

- parunits is a vector of units.

The second object, moutput has information on model output. It also has several components, the most important being out which is the output model matrix with rows referencing time and columns referencing model run. Other elements contain metadata: $t$ is the corresponding time vector, tunuts are time units, outname is the name of the modeled variable, and outunits are the corresponding units.

\section{Simple 1D example}

This and the following examples are based on running stilt version 1.3 .0 on $R$ version 3.3 .3 using a 3 Ghz Intel core i5 16GB $2400 \mathrm{MHz}$ DDR4 Macintosh 10.13.6 computer. The following versions were used for other required packages: fields (9.0), maps (3.3.0), spam (2.1-2), and dotCall64 (0.9-5). The results were observed to differ slightly according to the programming environment and the operating system.

We first consider interpolating a toy model dataset consisting of a simple time-series output for one parameter with a total of 21 parameter settings and 11 time points. The output of this simple model as a function of time $t$ and parameter $\theta$ is: $y=\sin (\theta)\left(1+2 t+t^{2}\right)$. The model is evaluated for $\theta=(0,1, \ldots, 20)$, and each run produces a time series for times $t=(0,1, \ldots, 10)$. First, we can plot model output for all parameters:

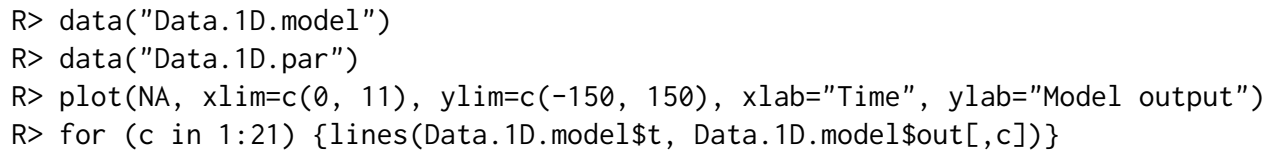

The output of this code is shown in Figure 1.

Now, we fit a Gaussian process emulator to these data. While we do not use any parameter covariances (par . reg=FALSE), we do use a linear time covariate (time. reg=TRUE). The optimization follows the default behavior of fixing the linear regressors at the multiple linear regression estimates. We select 100 as the starting values for both $\kappa$ and $\zeta$ because this leads to reasonable optimization results.

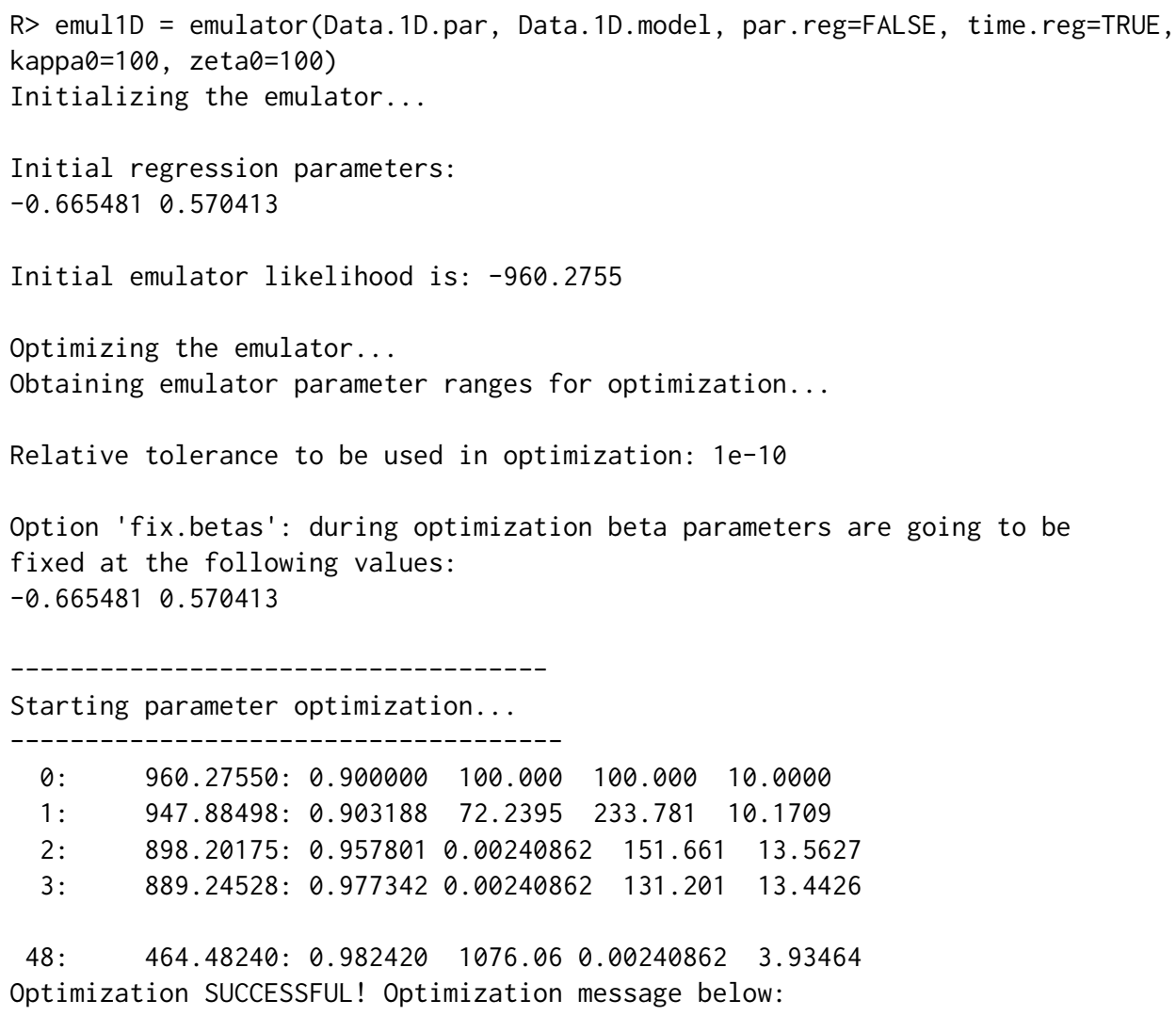




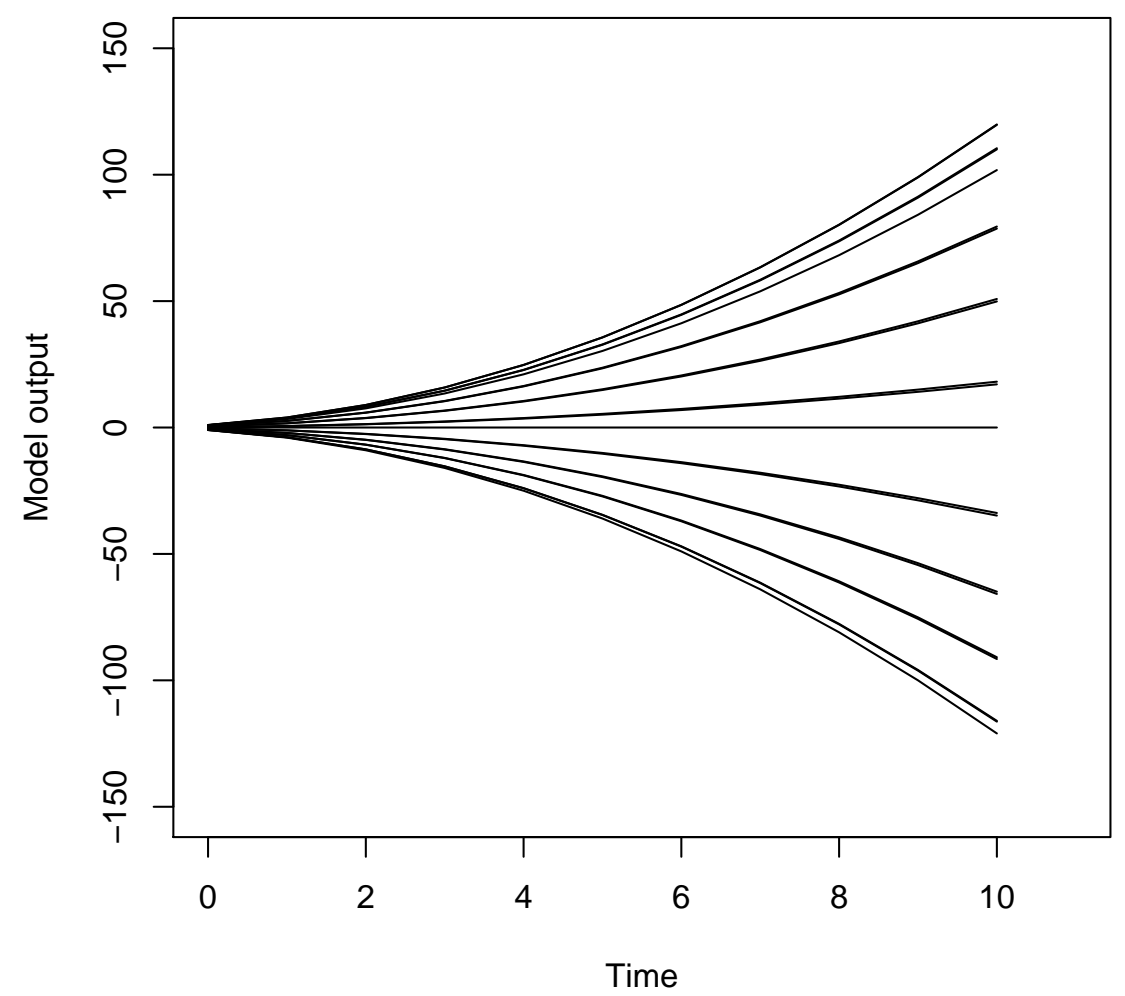

Figure 1: Sample time series output of the toy model for different parameter settings

relative convergence (4)

Final parameterss

rho kappa zeta phi

0.982420041076 .057145890 .002408623 .93464218

48 iterations were performed

Final likelihood $=-464.4824$

CAUTION! The optimization might only find a local minimum.

The package informs us of key emulator parameter settings, and of optimization results. (For brevity, only the start and the end of optimization process are shown above.) The list of parameters to be optimized is $\rho, \kappa, \zeta$, and $\phi_{1}$ in this case. The final emulator is a highly autocorrelated process with a large partial sill, but a very low nugget. The emulator object emul1D is a list with many components, with a secondary custom "emul" class. The components include information on the data used to fit the emulator, optimized emulator parameters, some settings used during optimization, time and parameter covariance matrices, and the inverse of the parameter covariance matrix. Now, we validate the emulator using one-at-a-time cross-validation for time index 9 (close to the end of the time series). Specifically, we remove each parameter setting from the ensemble one at a time, and use the emulator to predict the output at the excluded parameter setting, given the output at other parameter settings.

R> test.all(emul1D, 9)

The emulator mean prediction shows a remarkable accuracy at predicting actual model response: the two fall almost perfectly on the 1:1 line of a reliability diagram (Figure 2). The emulator prediction error is much less than $1 \%$ for almost all runs (Figure 2). Note that the emulator does not extrapolate beyond the ensemble parameter range.

The emulator predicts at new parameter settings using the function predict.emul. This is im- 

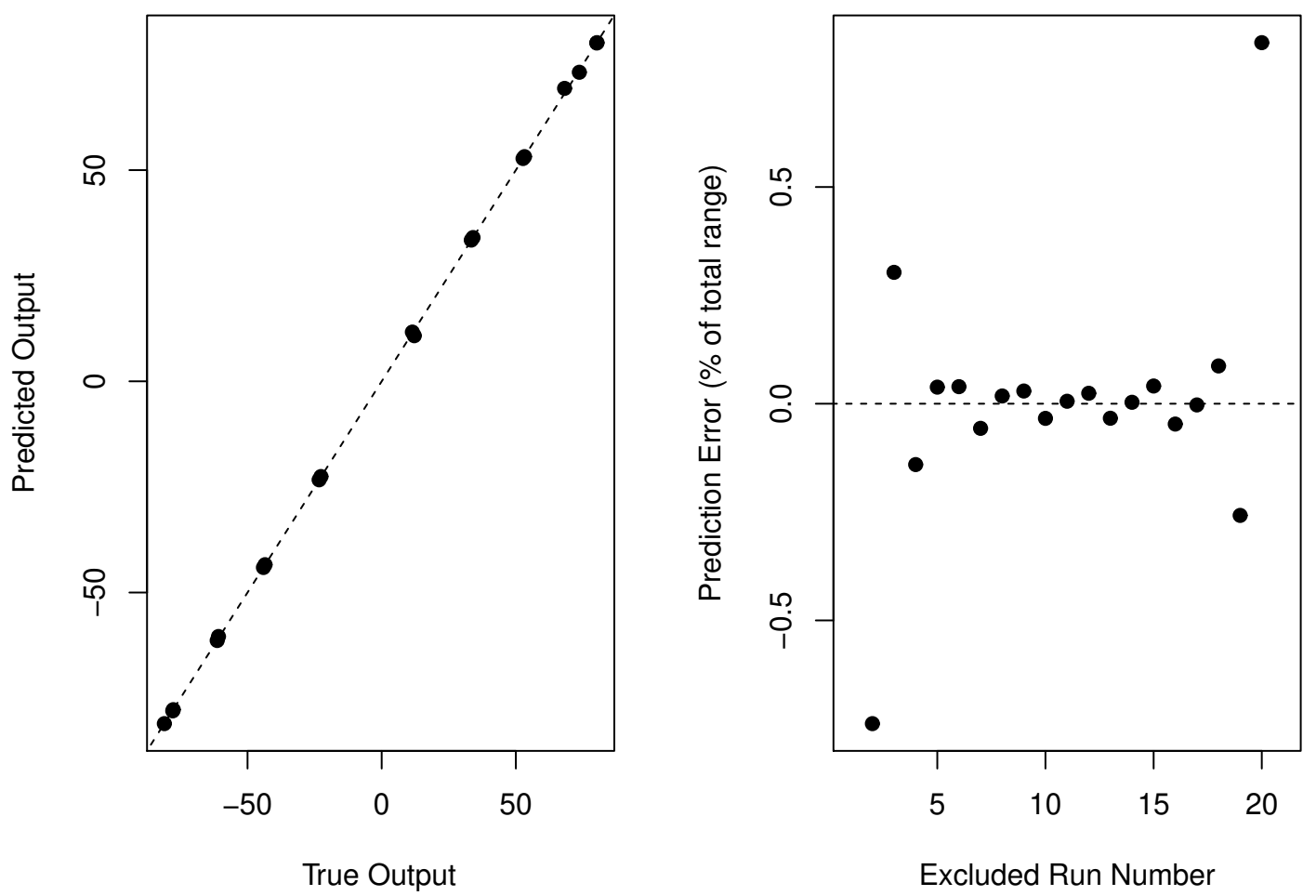

Figure 2: Toy example emulator one-at-a-time cross-validation results: (left) predicted vs. actual model output (black dots) and a 1:1 line; (right) relative prediction error as a function of the excluded model run number.

plemented using the generic S3 predict function that dispatches to the predict.emul() method for objects of class "emul". This function returns an object with components mean and covariance representing the mean and the covariance of the prediction.

\section{A more challenging test case: $2 \mathrm{D}$ Korean summer mean maximum temperature variabil- ity and change}

Next, we consider Korean summer mean maximum temperature output from 29 Coupled Model Intercomparison Project phase 5 (CMIP5) climate models (Taylor et al., 2012) for years 2081-2100 for the RCP8.5 forcing scenario (Moss et al., 2010). All of the models and calculations used here follow Shin et al. (2018), however here we exclude the model IPSL_CM5A_LR from the analysis. Of interest is the relationship between the present-day (years 1973-2005) sample red noise properties for annual temperature time-series, and the future mean maximum summer temperature changes in these climate models. Hence, each model $i$ is associated with $\boldsymbol{\theta}_{\boldsymbol{i}}=\left(\sigma_{i}, \rho_{i}\right)$, where $\sigma_{i}$ is sample innovation standard deviation, and $\rho_{i}$ is sample first-order autocorrelation. In this case, $m=2, p=29$, and $n=20$.

First, we load the relevant datasets and plot the temperature time series for all models.

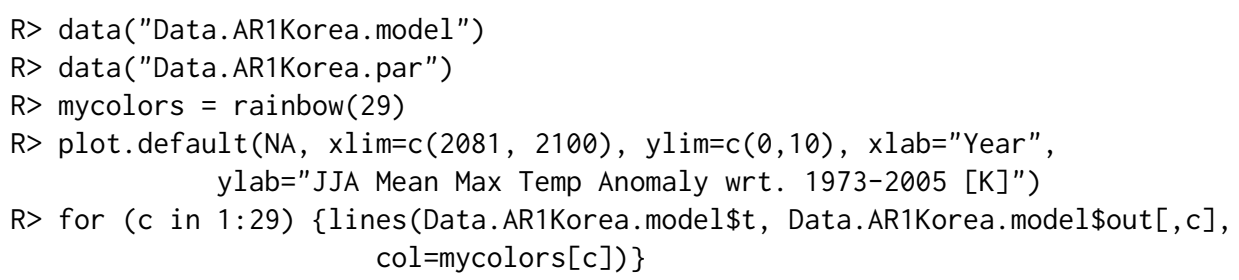

We note that the models show a considerable internal variability superimposed on a trend of slow warming (Figure 3). Next, we fit an emulator while also allowing for optimization of regression slopes 


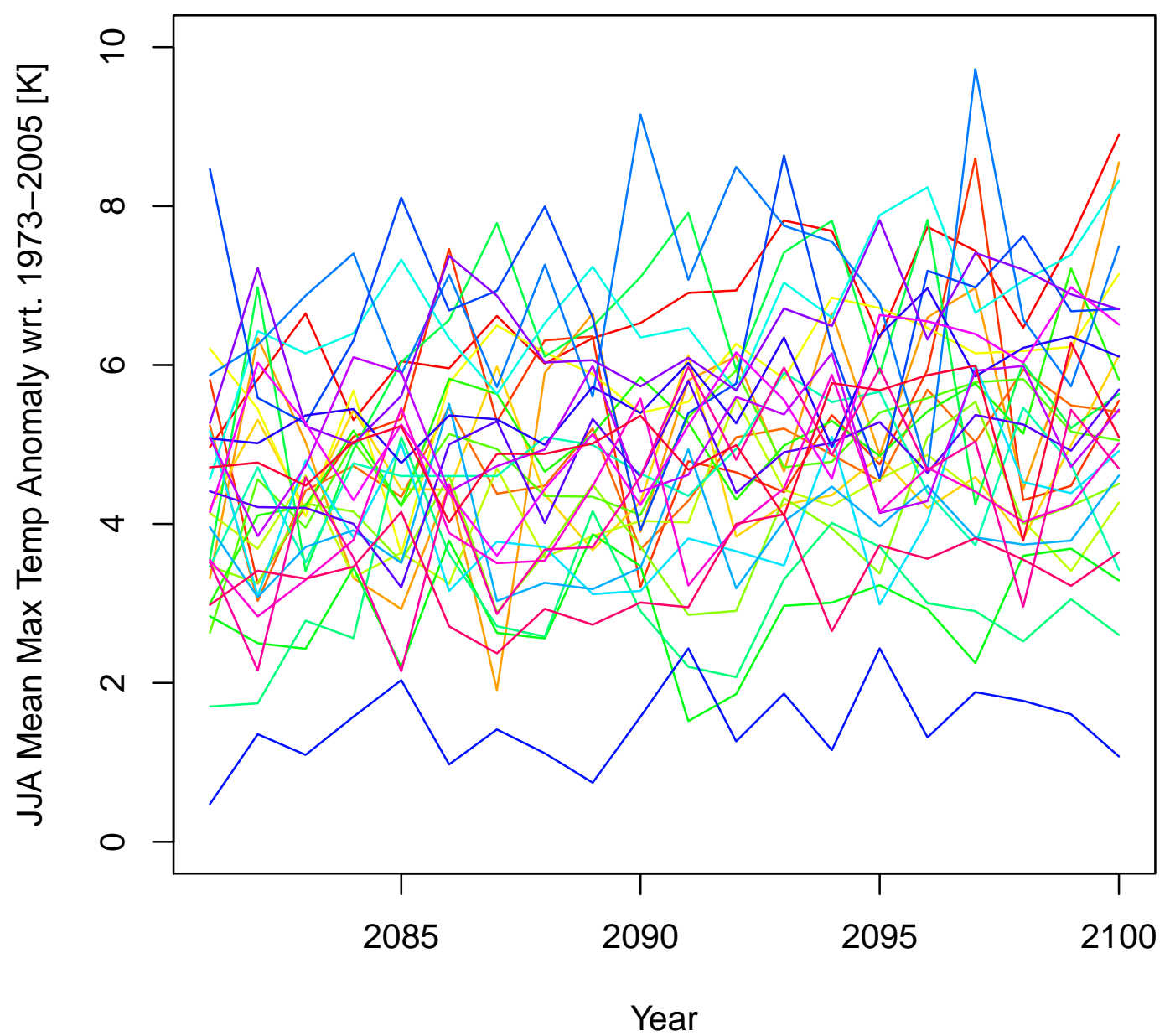

Figure 3: Korean summer mean maximum temperature change in years 2081-2100 with respect to period 1973-2005 for 29 CMIP5 climate models.

in innovation standard deviation and time. We also use a custom relative tolerance to illustrate how this parameter may be changed to fit user needs.

$\mathrm{R}>$ emul = emulator (Data.AR1Korea.par, Data.AR1Korea.model, par.reg=c (TRUE,

FALSE), time.reg=TRUE, $k a p p a \theta=1$, zeta $\emptyset=1$, myrel.tol $=1 \mathrm{E}-9$, fix. betas $=$ FALSE)

Initializing the emulator...

Initial regression parameters:

$\begin{array}{lll}-116.0626827 & 3.85448810 .0563853\end{array}$

Initial emulator likelihood is: $\mathbf{- 9 3 7 . 4 7 9}$

Optimizing the emulator...

obtaining emulator parameter ranges for optimization...

Relative tolerance to be used in optimization: $1 \mathrm{e}-09$ 


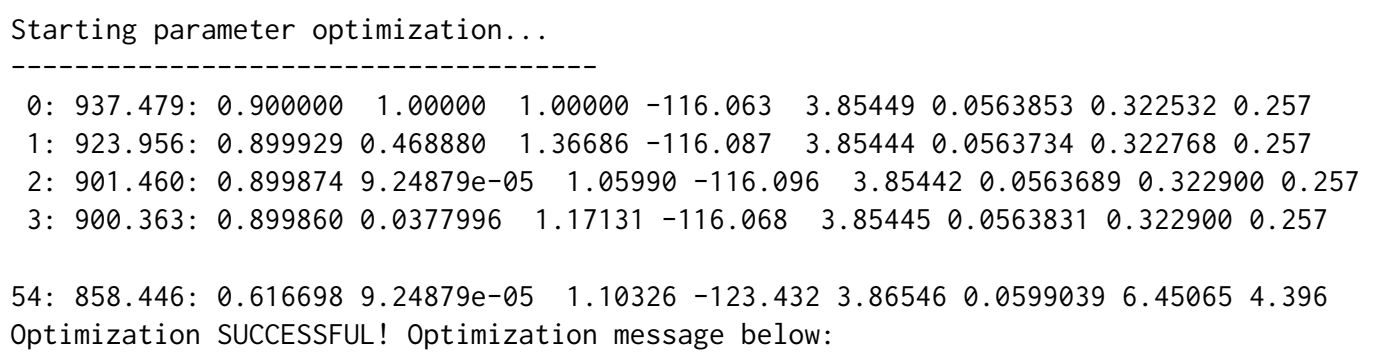

relative convergence (4)

Final parameterss

rho kappa zeta beta beta beta phi phi

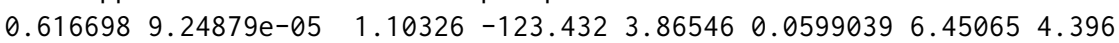

54 iterations were performed

Final likelihood $=-858.4463$

CAUTION! The optimization might only find a local minimum.

Note that we have formatted the output slightly to fit the page width.

We see that the initial multiple regression provides reasonable mean and parameter slopes. They are close to the final optimized results (fourth through sixth elements of the optimized parameter vector called "Final parameters" above). There is a considerable linear dependence on both time and innovation standard deviation. The non-linear part of the emulator has a strong random component, and a very weak Gaussian process component. This suggests no systematic dependence of the model output on the present-day autocorrelation. We can predict the temperature response at an arbitrary setting of the parameters using the predict function. We do this for $\sigma=1$ and $\rho=0$ :

$\mathrm{R}>$ pred $=\operatorname{predict}(\mathrm{emul}, \mathrm{c}(1,0))$

R> plot.default(NA, $x \lim =c(2081,2100), y l i m=c(3.5,7.5), x l a b=" Y e a r "$, ylab="JJA Mean Max Temp Anomaly wrt. 1973-2005 [K]")

R> lines (emul\$t.vec, pred\$mean)

R> lines(emul\$t.vec, pred\$mean + sqrt(diag(pred\$covariance)), col="brown")

R> lines(emul\$t.vec, pred\$mean - sqrt(diag(pred\$covariance)), col="brown")

The mean vector of the prediction is the mean component of pred, and the variance vector is composed of the diagonal entries of covariance. Figure 4 shows the predicted response, with the associated 1-std uncertainty. We note the linearity of the warming in time. This illustrates the ability of the emulator to identify fluctuations of temperature in the models around the linear trend as random, and not to include them into the emulated response.

\section{An even more challenging test case: Five-dimensional ice sheet model output}

Next, we consider a 5D emulator for the SICOPOLIS ice sheet model output (Greve, 1997; Greve et al., 2011) for Greenland ice mass loss relative to the year 2003. This is a perturbed parameter 100-member ensemble which varies five model parameters: flow enhancement factor, basal sliding factor, geothermal heat flux, snow positive degree-day (PDD) factor, and ice PDD factor. The future forcing scenario is that of a gradual temperature increase stabilizing at approximately $5 \mathrm{~K}$ warmer than present (Applegate et al., 2012). Output is available annually between years 1840 and 2500 .

We load relevant data and fit an emulator to the ensemble using all five parameters and time as covariates. For computational expediency, we fix slope parameters at their multiple regression estimates.

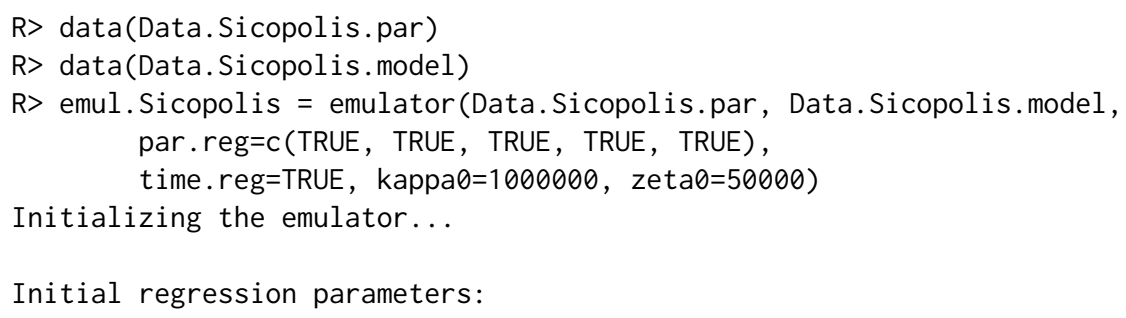




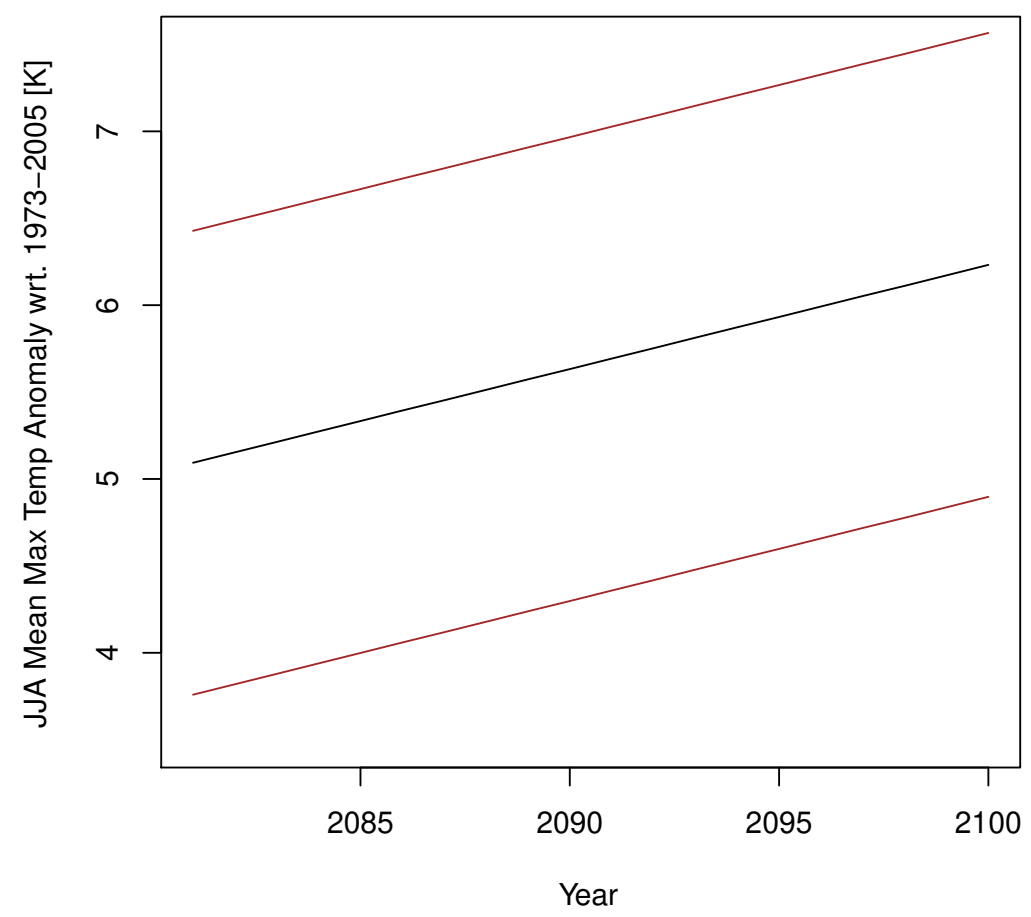

Figure 4: Emulated Korean summer mean maximum temperature change projections with respect to 1973-2005 at $\sigma=1 \mathrm{~K}$ and $\rho=0$. Solid and dashed lines: mean response \pm standard errors.

$5154878.375171 .401 \quad 8590.267 \quad-182.659 \quad 49920.313 \quad 1300.488-2725.378$

Initial emulator likelihood is: -10782007

Optimizing the emulator...

Obtaining emulator parameter ranges for optimization...

Relative tolerance to be used in optimization: $1 \mathrm{e}-10$

Option 'fix.betas': during optimization beta parameters are going to be fixed at the following values:

$5154878.375171 .401 \quad 8590.267 \quad-182.65949920 .313 \quad 1300.488$-2725.378

Starting parameter optimization...

\begin{tabular}{|c|c|c|c|c|c|c|c|c|c|}
\hline $0:$ & 10782007.: & 0.900 & $1.00 \mathrm{e}+06$ & 50000.0 & .97305 & .84502 & 19.9205 & $1.98380 \quad 7$ & .45 \\
\hline 1: & 672836.29: & 1.00 & $3.29 e+07$ & $7.05408 \mathrm{e}+07$ & 2.83691 & 8.68834 & $4 \quad 27.1292$ & 2.24728 & 0.00149 \\
\hline 2: & 672613.27: & 0.999 & $3.29 \mathrm{e}+07$ & $7.05341 \mathrm{e}+07$ & 2.83691 & 8.68834 & $4 \quad 27.1292$ & 2.24728 & 0.0537 \\
\hline 3: & 667210.63: & 0.997 & $3.20 \mathrm{e}+07$ & $7.08502 \mathrm{e}+07$ & 2.86488 & 38.69561 & 27.3808 & $\begin{array}{l}32.26410 \\
\end{array}$ & 14.54 \\
\hline & 485611.17: & 0.99 & $5.83 \epsilon$ & 41529.0 & 13.4764 & 20.1003 & $3 \quad 199.578$ & 5.72313 & 10.9 \\
\hline
\end{tabular}

Optimization SUCCESSFUL! Optimization message below:

relative convergence (4)

Final parameters

rho kappa zeta phi phi phi phi phi

$0.9999895829746 .77013541528 .99333913 .476403 \quad 20.100261 \quad 199.578404 \quad 5.723128 \quad 10.901509$ 
79 iterations were performed

Final likelihood $=-485611.2$

CAUTION! The optimization might only find a local minimum.

The emulator takes roughly 3 minutes to fit on a $3 \mathrm{Ghz}$ Intel core i5 16GB $2400 \mathrm{MHz}$ DDR4 Macintosh computer. The final emulator is very smooth as evidenced by the relatively high range parameters compared to the ensemble parameter range, and has an extremely low nugget compared to the partial sill parameter.

Next, we perform cross-validation of the emulator for the entire time series. We withhold three ensemble members, and predict at withheld parameters using the model output at the remaining 97 parameter settings.

R> test.csv(emul.Sicopolis, num.test=3, plot.std=TRUE, theseed=13241240)

Predicting for run number: 3

Predicting for run number: 26

Predicting for run number: 93

Here, we have specified a random seed, and the output tells us which model runs were excluded. Alternatively, we can select the runs to withhold via the test. runind argument. We present the results in Figure 5. The emulator has remarkable skill at recovering the output of the withheld models.

Now, we withhold more runs and perform a more systematic analysis of emulator behavior:

$\mathrm{R}>$ mytest $=$ test. $\operatorname{csv}($ emul.Sicopolis, num.test=10, plot.std=FALSE, theseed=13241240, make.plot=FALSE)

Predicting for run number: 3

Predicting for run number: 7

Predicting for run number: 26

Predicting for run number: 34

Predicting for run number: 37

Predicting for run number: 43

...Prediction error. Likely because prediction parameters are out of bounds

Predicting for run number: 91

Predicting for run number: 93

Predicting for run number: 99

Predicting for run number: 100

NOTE: 1 prediction points were omitted

R> cat ("95\% CI coverage:", mytest\$coverage, "\n")

95\% CI coverage: 0.9768028

Note that stilt does not extrapolate beyond the parameter range of the ensemble. Since one of the parameters is at its maximum among the ensemble for the $43^{r d}$ run, this run is skipped during the cross-validation. We disable the plotting since our main interest here is empirical coverage of the $95 \%$ prediction interval. The coverage (0.977) is relatively close to the ideal theoretical value of 0.95 , which suggests that the emulator is relatively well calibrated.

We finish by plotting the response surface of the emulator for parameters 4 (snow PDD factor), and 5 (ice PDD factor). We fix flow enhancement factor, basal sliding factor, and geothermal heat flux at values of 3.0, $10.0 \mathrm{~m} \mathrm{y}^{-1} \mathrm{~Pa}^{-1}$, and $45.0 \mathrm{~m} \mathrm{~W} \mathrm{~m}^{-2}$, respectively.

R> rsurface.plot (emul.Sicopolis, parind $=c(4,5)$, parvals $=c(3,10,45, N A, N A)$,

tind $=600, \mathrm{n} 1=10, \mathrm{n} 2=10$ )

We look at the $600^{\text {th }}$ time index (year 2439), where $\mathrm{n} 1$ and $\mathrm{n} 2$ are the number of grid points to use in the $x$ and $y$ directions, respectively. Figure 6 shows the response surface. A monotonic positive relationship exists between the ice mass anomaly as a function of the snow PDD factor across most of the ice PDD factor range. However, the relationship between the ice mass loss and the ice PDD factor appears to be non-monotonic.

\section{Concluding remarks}

Here, we present stilt - a package for simplified Gaussian process emulation. The package is designed for emulation of time series model output in parameter space, although it can be applied for kriging more generally. The focus is on simplicity, so the package could be easily applied to many challenging 


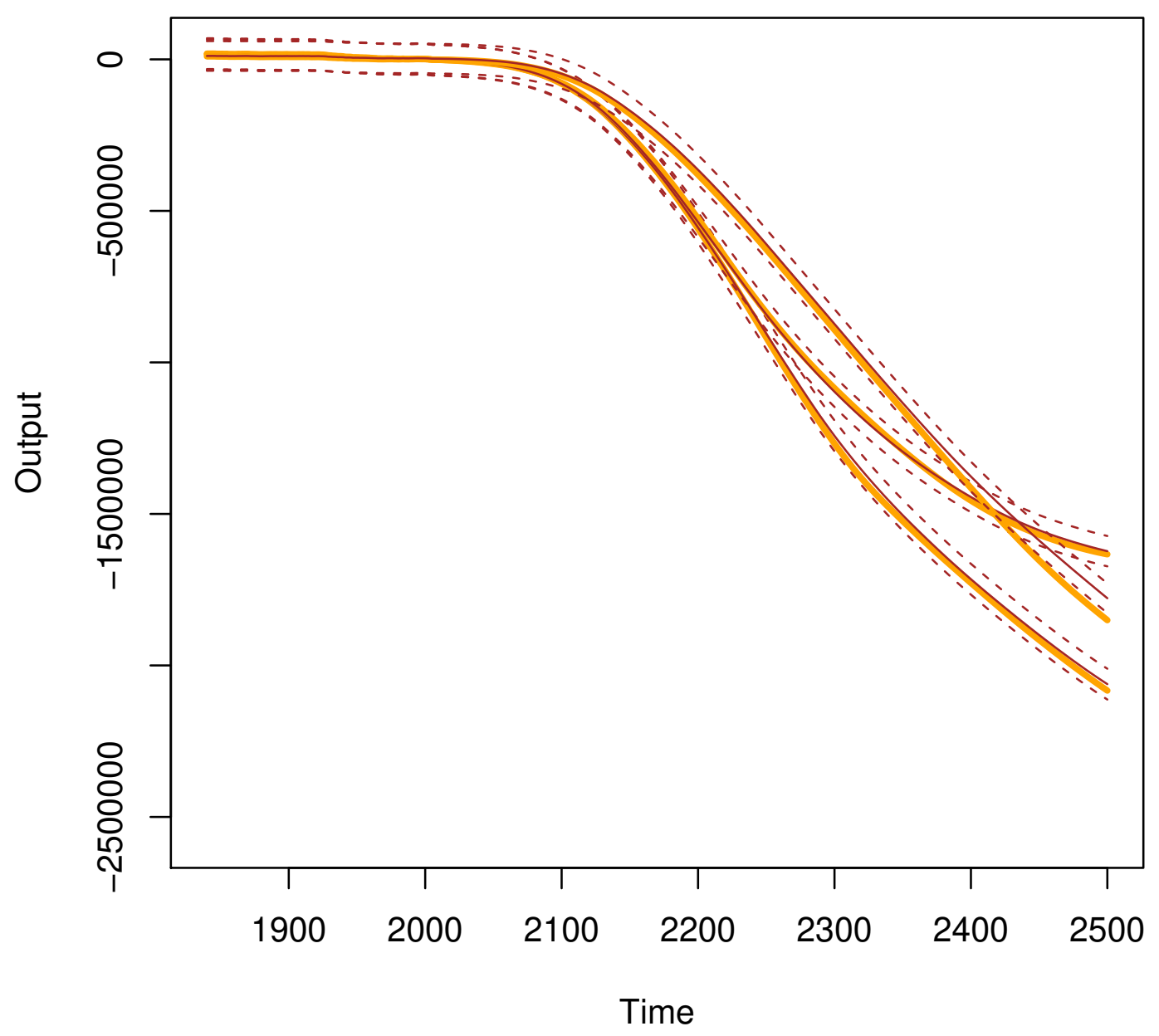

Figure 5: Cross-validation of Greenland Ice Sheet anomaly with respect to year 2003 [Gt] from the SICOPOLIS ensemble. Thick beige lines: actual model output. Brown lines: emulator predictions with standard error confidence intervals.

problems by users outside of the statistics research community. The streamlined emulator fitting and prediction means the package is also useful pedagogically in demonstrating the Gaussian process emulation. We assume separability in space and time for the Gaussian process. This allows us to reduce computational burden using matrix algebra, and makes it possible to apply the package to moderately large datasets, especially in the time dimension. We showcase package capabilities on three examples which differ in the amount of parameters in the model ensemble. Specifically, we use a $1 \mathrm{D}$ toy dataset, a 2D dataset of Korean summer mean maximum temperatures, and the 5D SICOPOLIS ice sheet model output. Using cross-validation functions, we show the capability of the emulator to predict model response at excluded parameter values in up to five dimensions. We demonstrate the capacity to visualize estimated $2 \mathrm{D}$ model response surfaces. The package can be useful when computational resources are limited, and a relatively fast statistical approximator is required for a complex model across a range of parameter space. Some limitations of the package are homoskedasticity, separability, and the fixed covariance structure (e.g., AR(1) and the squared exponential function). We choose the AR(1) model because it is applicable to data in diverse scientific fields (Hasselmann, 1976; Keller and McInerney, 2008; Li, 2011; Olson et al., 2013). The disadvantage is that such an emulator may not handle seasonal or periodic effects that may be present in computer model output. Extending the package to account for heteroskedasticity (e.g., hetGP package, Binois and Gramacy, 2017) should be considered in future work. 


\section{Output}

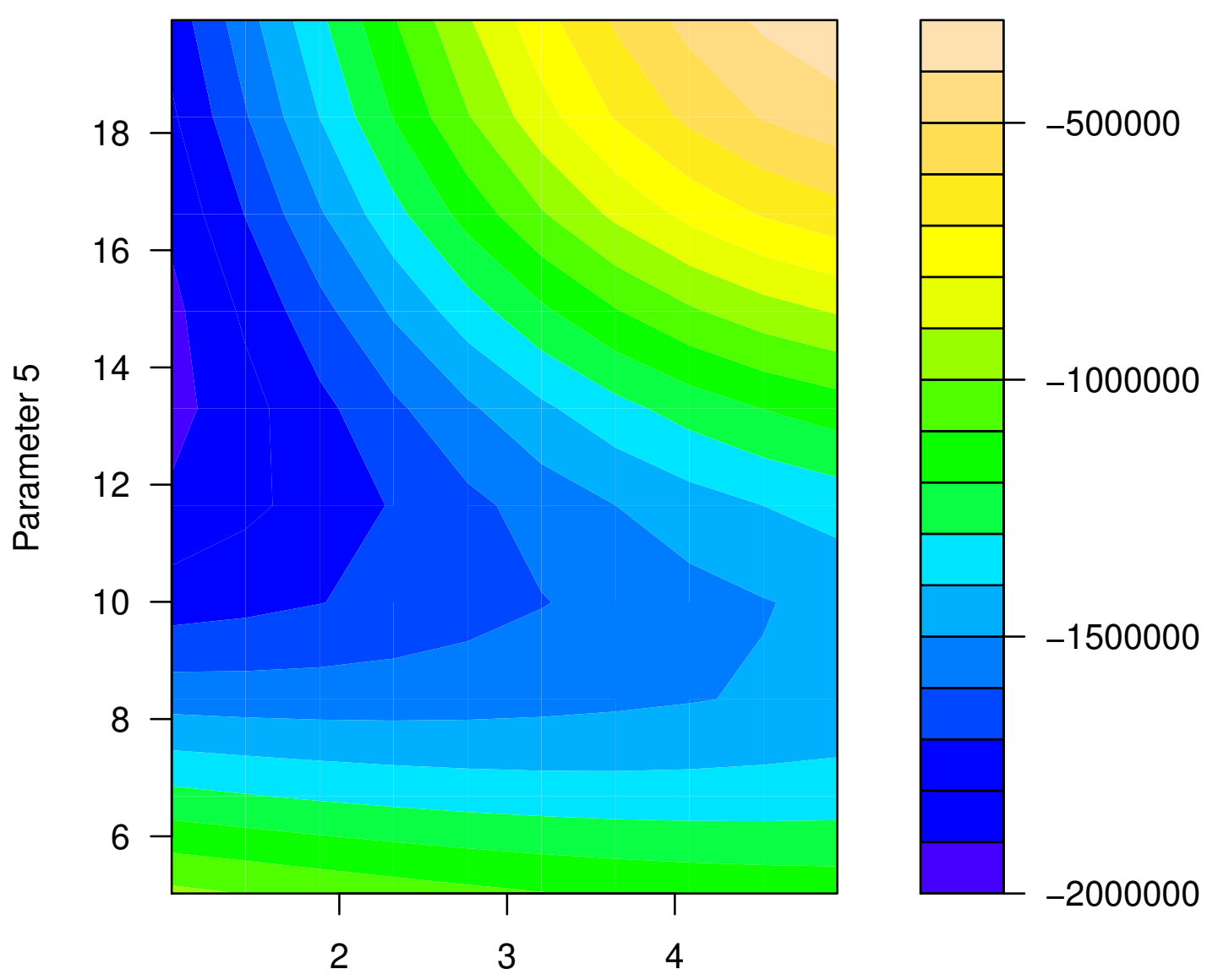

\section{Parameter 4}

Figure 6: Response surface of SICOPOLIS Greenland Ice Sheet anomaly with respect to year 2003 [Gt] for year 2439 as a function of parameter 4 (snow PDD factor, $\mathrm{mm} \mathrm{day}^{-1} \mathrm{~K}^{-1}$ ) and parameter 5 (ice PDD factor, $\mathrm{mm}_{\text {day }}{ }^{-1} \mathrm{~K}^{-1}$ )

\section{Acknowledgments}

For their roles in producing, coordinating, and making available the CMIP5 model output, we acknowledge the climate modeling groups, the World Climate Research Programme's (WCRP) Working Group on Coupled modeling (WGCM), and the Global Organization for Earth System Science Portals (GO-ESSP). We thank Jong-Soo Shin for help with extracting Korean temperature output, and Patrick Applegate for sharing the SICOPOLIS ice sheet model output. We acknowledge financial support from National Research Foundation of Korea (NRF-2009-0093069, NRF-2018R1A5A1024958), and from the Institute for Basic Science (project code IBS-R028-D1). This work was also co-supported by the National Science Foundation through the Network for Sustainable Climate Risk Management (SCRiM) under NSF cooperative agreement GEO-1240507 and the Penn State Center for Climate Risk Management. Any opinions, findings, and conclusions or recommendations expressed in this material are those of the authors and do not necessarily reflect the views of the National Science Foundation, or any other foundation or entity. 


\section{Appendix}

This Appendix describes the technique to reduce computational cost when evaluating the likelihood (Equation 8). In its original form, it involves a computationally expensive inverse of an $n p \times n p$ matrix. Consider a $p \times n$ matrix $\mathbf{C}$, where $\mathbf{Y}-\boldsymbol{\mu}_{\beta}=\operatorname{vec}(\mathbf{C})$, and the vec operation stacks columns of a matrix into a column vector, from left to right (Rougier, 2008). Using properties of Kronecker products (Golub and Van Loan, 1996), of the vec operator (Magnus and Neudecker, 2007), and other matrix algebra:

$$
\begin{aligned}
\left(\mathbf{Y}-\boldsymbol{\mu}_{\boldsymbol{\beta}}\right)^{T} \Sigma^{-1}\left(\mathbf{Y}-\boldsymbol{\mu}_{\boldsymbol{\beta}}\right) & =(\operatorname{vec}(\mathbf{C}))^{T}\left[\Sigma_{t}^{-1} \otimes \Sigma_{\theta}^{-1}\right] \operatorname{vec}(\mathbf{C}) \\
& =(\operatorname{vec}(\mathbf{C}))^{T}\left[\left(\Sigma_{t}^{-1}\right)^{T} \otimes \Sigma_{\theta}^{-1}\right] \operatorname{vec}(\mathbf{C}) \\
& =(\operatorname{vec}(\mathbf{C}))^{T} \operatorname{vec}\left(\Sigma_{\theta}^{-1} \mathbf{C} \Sigma_{t}^{-1}\right) \\
& =\operatorname{sum}\left[\mathbf{C} *\left(\Sigma_{\theta}^{-1} \mathbf{C} \Sigma_{t}^{-1}\right)\right]
\end{aligned}
$$

where $*$ is the Hadamard product. This reduces the dimension of matrices that are inverted to $p \times p$ and $n \times n$, thus substantially reducing computational burden. We further note that both matrices $\Sigma_{\theta}$ and $\Sigma_{t}$ are positive definite (Wicklin, 2013, and others). Thus, the inverses can be found through the Cholesky decomposition of $\Sigma_{\theta}=\mathbf{R}_{\theta}^{T} \mathbf{R}_{\theta}$, where $\mathbf{R}_{\theta}$ (called Cholesky factor) is an upper triangular matrix; and similarly for $\Sigma_{t}$.

Additionally, determinant computations can be simplified considerably (Gentle, 2007):

$$
|\Sigma|=\left|\Sigma_{t} \otimes \Sigma_{\theta}\right|=\left|\Sigma_{t}\right|^{p}\left|\Sigma_{\theta}\right|^{n} .
$$

Thus, we only need to evaluate the determinants of the individual covariance matrices. Moreover, since $\Sigma_{\theta}$ and $\Sigma_{t}$ are positive definite, $\left|\Sigma_{\theta}\right|=\prod_{i=1}^{p} r_{\theta, i i}^{2}$, where $r_{\theta, i i}$ are diagonal elements of the Cholesky factor $\mathbf{R}_{\theta}$. Similar calculations can be performed for $\left|\Sigma_{t}\right|$.

\section{Bibliography}

P. J. Applegate, N. Kirchner, E. J. Stone, K. Keller, and R. Greve. An assessment of key model parametric uncertainties in projections of Greenland ice sheet behavior. Cryosphere, 6(3):589-606, 2012. URL https://doi.org/10.5194/tc-6-589-2012. [p217]

K. S. Bakar and S. K. Sahu. spTimer: Spatio-temporal Bayesian modeling using R. Journal of Statistical Software, 63(15):1-32, 2015a. URL https://doi .org/10.18637/jss. v063.i15. [p209]

K. S. Bakar and S. K. Sahu. spTimer: Spatio-Temporal Bayesian Modeling Using R, 2015b. R package version 2.0-1. [p209]

K. S. Bhat, M. Haran, R. Olson, and K. Keller. Inferring likelihoods and climate system characteristics from climate models and multiple tracers. Environmetrics, 23(4):345-362, 2012. URL https://doi . org/10.1002/env. 2149. [p209,210]

M. Binois and R. B. Gramacy. hetGP: Heteroskedastic Gaussian Process Modeling and Design under Replication, 2017. URL https://CRAN. R-project.org/package=hetGP. R package version 1.0.1. [p220]

W. Chang, P. J. Applegate, M. Haran, and K. Keller. Probabilistic calibration of a Greenland ice sheet model using spatially resolved synthetic observations: Toward projections of ice mass loss with uncertainties. Geoscientific Model Development, 7(5):1933-1943, 2014a. ISSN 1991-959X. URL https://doi.org/10.5194/gmd-7-1933-2014. [p209]

W. Chang, M. Haran, R. Olson, and K. Keller. Fast dimension-reduced climate model calibration and the effect of data aggregation. Annals of Applied Statistics, 8(2):649-673, 2014b. ISSN 1932-6157. URL https://doi.org/10.1214/14-AOAS733. [p209,211]

N. Cressie. Statistics for Spatial Data. Wiley Series in Probability and Mathematical Statistics, New York, U.S.A, 1993. URL https://doi .org/10.1002/9781119115151. [p210]

N. Cressie and G. Johannesson. Fixed rank kriging for very large spatial data sets. Journal of the Royal Statistical Society. Series B: Statistical Methodology, 70(1):209-226, 2008. URL https://doi .org/10. $1111 / j .1467-9868.2007 .00633 . x$. [p210]

G. M. Dancik. mlegp: Maximum Likelihood Estimates of Gaussian Processes, 2013. URL https: //CRAN. Rproject. org/package=mlegp. R package version 3.1.4. [p209, 211] 
G. M. Dancik and K. S. Dorman. mlegp: Statistical analysis for computer models of biological systems using R. Bioinformatics, 24(17):1966-1967, 2008. URL https://doi .org/10.1093/bioinformatics/ btn329. [p209, 211]

D. Drignei, C. E. Forest, and D. Nychka. Parameter estimation for computationally intensive nonlinear regression with an application to climate modeling. Annals of Applied Statistics, 2(4):1217-1230, 2008. URL https://doi.org/10.1214/08-A0AS210. [p209]

A. O. Finley, S. Banerjee, and A. E.Gelfand. spBayes for large univariate and multivariate pointreferenced spatio-temporal data models. Journal of Statistical Software, 63(13):1-28, 2015. URL https://doi.org/10.18637/jss.v063.i13. [p209]

D. M. Gay. Usage summary for select optimization routines. Computing Science Technical Report 153. Technical report, AT\&T Bell Laboratories, Murray Hill, New Jersey, U. S. A., 1990. [p212]

J. E. Gentle. Matrix Algebra. Theory, Computations, and Applications in Statitics. Springer, New York, U.S.A, 2007. URL https://doi.org/10.1007/978-0-387-70873-7. [p222]

G. H. Golub and C. F. Van Loan. Matrix Computations. John Hopkins University Press, 3 edition, 1996. [p212, 222]

R. Greve. Application of a polythermal three-dimensional ice sheet model to the Greenland ice sheet: Response to steady-state and transient climate scenarios. Journal of Climate, 10(5):901-918, MAY 1997. [p217]

R. Greve, F. Saito, and A. Abe-Ouchi. Initial results of the SeaRISE numerical experiments with the models SICOPOLIS and IcIES for the Greenland ice sheet. Annals of Glaciology, 52(58):23-30, 2011. URL https://doi .org/10.3189/172756411797252068. [p217]

B. Gräler, E. Pebesma, and G. Heuvelink. Spatio-Temporal Interpolation using gstat. The R Journal, 8 (1):204-218, 2016. [p209]

J. Hansen, R. Ruedy, M. Sato, and K. Lo. Global surface temperature change. Reviews of Geophysics, 48 (4), 2010. URL https://doi.org/10.1029/2010RG000345. [p210]

K. Hasselmann. Stochastic climate models part I. Theory. Tellus, 28(6):473-485, 1976. ISSN 2153-3490. URL https://doi.org/10.3402/tellusa.v28i6.11316. [p211,220]

D. Higdon, J. Gattiker, B. Williams, and M. Rightley. Computer model calibration using highdimensional output. Journal of the American Statistical Association, 103(482):570-583, 2008. URL https://doi.org/10.1198/016214507000000888. [p209,211]

S. Hirahara, M. Ishii, and Y. Fukuda. Centennial-scale sea surface temperature analysis and its uncertainty. Journal of Climate, 27(1):57-75, 2014. URL https://doi.org/10.1175/JCLI-D-1200837. 1. [p210]

P. B. Holden, N. R. Edwards, K. I. C. Oliver, T. M. Lenton, and R. D. Wilkinson. A probabilistic calibration of climate sensitivity and terrestrial carbon change in GENIE-1. Climate Dynamics, 35(5): 785-806, 2010. URL https://doi.org/10.1007/s00382-009-0630-8. [p209]

D. A. Jones, W. Wang, and R. Fawcett. High-quality spatial climate data-sets for Australia. Australian Meteorological and Oceanographic Journal, 58(4):233-248, 2009. URL https://doi .org/10. 22499/2. 5804.003. [p210]

K. Keller and D. McInerney. The dynamics of learning about a climate threshold. Climate Dynamics, 30 (2-3):321-332, 2008. URL https://doi .org/10.1007/s00382-007-0290-5. [p211, 220]

M. Kennedy and A. O'Hagan. Bayesian calibration of computer models. Journal of the Royal Statistical Society Series B - Statistical Methodology, 63(3):425-450, 2001. ISSN 1369-7412. URL https://doi. org/10.1111/1467-9868.00294. [p209]

Z. Li. Evaluating GDP forecasting models for Korea. Technical report, International Monetary Fund, 2011. [p220]

J. Magnus and H. Neudecker. Matrix Differential Calculus with Applications in Statistics and Econometrics. John Wiley \& Sons, 3rd edition, 2007. [p222] 
R. H. Moss, J. A. Edmonds, K. A. Hibbard, M. R. Manning, S. K. Rose, D. P. Van Vuuren, T. R. Carter, S. Emori, M. Kainuma, T. Kram, G. A. Meehl, J. F. B. Mitchell, N. Nakicenovic, K. Riahi, S. J. Smith, R. J. Stouffer, A. M. Thomson, J. P. Weyant, and T. J. Wilbanks. The next generation of scenarios for climate change research and assessment. Nature, 463(7282):747-756, 2010. URL https://doi.org/10.1038/nature08823. [p215]

R. Olson, R. Sriver, M. Goes, N. M. Urban, H. D. Matthews, M. Haran, and K. Keller. A climate sensitivity estimate using Bayesian fusion of instrumental observations and an Earth system model. Journal of Geophysical Research Atmospheres, 117(4), 2012. URL https://doi .org/10.1029/2011 JD016620. [p209]

R. Olson, R. Sriver, W. Chang, M. Haran, N. M. Urban, and K. Keller. What is the effect of unresolved internal climate variability on climate sensitivity estimates? Journal of Geophysical Research Atmospheres, 118(10):4348-4358, 2013. URL https: //doi .org/10.1002/jgrd. 50390. [p209, 211, 220]

R. Olson, W. Chang, K. Keller, and M. Haran. stilt: Separable Gaussian Process Interpolation (Emulation), 2017. URL https://CRAN.R-project.org/package=stilt. R package version 1.2.0. [p210]

C. E. Rasmussen and C. K. I. Williams. Gaussian Processes for Machine Learning. The MIT Press, 2006. URL http: //www. gaussianprocess.org/gpml/chapters/RW.pdf. [p209, 210, 212]

J. Rougier. Efficient emulators for multivariate deterministic functions. Journal of Computational and Graphical Statistics, 17(4):827-843, 2008. URL https://doi .org/10.1198/106186008X384032. [p209, 210, 211, 212, 222]

M. Schlather, A. Malinowski, P. J. Menck, M. Oestin, and K. Strokorb. Analysis, simulation and prediction of multivariate random fields with package randomfields. Journal of Statistical Software, 63(8):1-25, 2015. URL https://doi.org/10.18637/jss.v063.i08. [p209]

D. M. H. Sexton, J. M. Murphy, M. Collins, and M. J. Webb. Multivariate probabilistic projections using imperfect climate models part I: Outline of methodology. Climate Dynamics, 38(11-12):2513-2542, 2012. URL https://doi .org/10.1007/s00382-011-1208-9. [p209]

J. Shin, R. Olson, and S.-I. An. Projected heat wave characteristics over the Korean Peninsula during the twenty-first century. Asia-Pacific Journal of Atmospheric Sciences, 54(1):53-61, 2018. URL https: //doi.org/10.1007/s13143-017-0059-7. [p215]

B. J. Smith, J. Yan, and M. K. Cowles. Unified geostatistical modeling for data fusion and spatial heteroskedasticity with R package ramps. Journal of Statistical Software, 25(10):1-21, 2008. URL https://doi.org/10.18637/jss.v025.i10. [p209]

M. L. Stein. Interpolation of Spatial Data: Some Theory for Kriging. Springer Science+Business Media, New York, U.S.A, 1999. URL https://doi .org/10.1007/978-1-4612-1494-6. [p209, 210]

K. E. Taylor, R. J. Stouffer, and G. A. Meehl. An overview of CMIP5 and the experiment design. Bulletin of the American Meteorological Society, 93(4):485-498, 2012. URL https://doi .org/10.1175/BAMS-D11-00094.1. [p215]

R. Wicklin. Simulating Data with SAS. The SAS Institute, 2013. [p222]

Roman Olson

Department of Atmospheric Sciences

Yonsei University

529A Sciences Building, 50 Yonsei-ro

Sinchon-dong, Seodaemun-gu, Seoul, 03722, South Korea

And:

Center for Climate Physics

Institute for Basic Science

Room 1111, Tonghapgigyegwan Building

Busandaehak-ro 63 beon-gil 2

Geumjeong-gu, Busan, 46241

South Korea

And:

Pusan National University, Busan, South Korea, 46241

romanolson@pusan.ac. kr 
Kelsey L. Ruckert

Penn State University

Earth and Environmental Systems Institute

320c EES Building

University Park, PA, 16802, United States of America

kIr324@psu.edu

Won Chang

Division of Statistics and Data Science

Department of Mathematical Sciences

University of Cincinatti

5516 French Hall, 2815 Commons Way

Cincinatti, OH, 45221-0025, United States of America

won. chang@uc . edu

Klaus Keller

Department of Geosciences

Penn State University

436 Deike Building

University Park, PA, 16802, United States of America

kzk10@psu.edu

Murali Haran

Department of Statistics

Penn State University

326 Thomas Building

University Park, PA, 16802, United States of America

mharan@stat.psu.edu

Soon-Il An

Department of Atmospheric Sciences

Yonsei University

538 Sciences Building, 50 Yonsei-ro

Sinchon-dong, Seodaemun-gu, Seoul, 03722, South Korea

sian@yonsei.ac.kr 